Dieses Dokument ist eine Zweitveröffentlichung (Verlagsversion) /

This is a self-archiving document (published version)

„Dieser Beitrag ist mit Zustimmung des Rechteinhabers aufgrund einer (DFG-geförderten) Allianzbzw. Nationallizenz frei zugänglich."

This publication is openly accessible with the permission of the copyright owner. The permission is granted within a nationwide license, supported by the German Research Foundation (abbr. in German DFG).

https://www.nationallizenzen.de
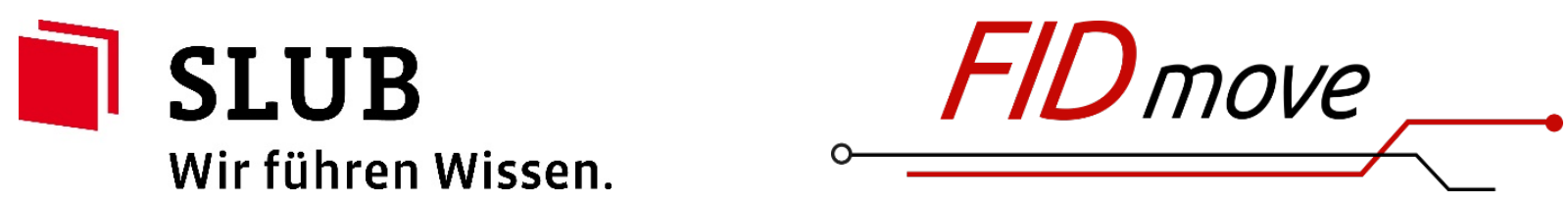


\title{
Effect of clocking on unsteady rotor blade loading in a low-speed axial compressor at design and off-design operating conditions
}

\author{
H-X Jia ${ }^{1}$, G Xi1 ${ }^{1 *}$, L Müller ${ }^{2}$, R Mailach ${ }^{2}$, and K Vogeler ${ }^{2}$ \\ ${ }^{1}$ School of Energy and Power Engineering, Xi'an Jiaotong University, Xi'an, People's Republic of China \\ ${ }^{2}$ Institute for Fluid Mechanics, Technische Universität Dresden, Dresden, Germany
}

The manuscript was received on 24 July 2007 and was accepted after revision for publication on 24 April 2008.

DOI: 10.1243/09544100JAERO262

\begin{abstract}
This paper presents the results of stator clocking investigations at a design point and an operating point near the stability limit in a low-speed research compressor (LSRC).

The unsteady flow field of the LSRC at several clocking configurations was investigated using a three-dimensional unsteady, viscous solver. The unsteady pressure on the rotor blades at midspan (MS) was measured using time-resolving piezoresistive miniature pressure transducers.

The effect of clocking on the unsteady pressure fluctuation at MS on the rotor blades is discussed for different operating points.

Based on the unsteady profile pressures, the blade pressure forces were calculated. The peakto-peak amplitudes of the unsteady blade pressure forces are presented and analysed for different clocking positions at both the design point and the operating point near the stability limit of the compressor.
\end{abstract}

Keywords: axial compressor, rotor-stator-interaction, periodic unsteady blade pressure fluctuation, unsteady blade pressure force

\section{INTRODUCTION}

The flow in multi-stage turbomachines is inherently unsteady because of the relative motion between the rotor and stator blade rows. This causes aerodynamic interactions of the wakes from the upstream blade row and the potential flow field from the downstream blade row. The continuing requirement to improve the durability and performance of turbomachines has motivated designers to deeply understand the unsteady interaction mechanisms between the rotor and stator blade rows.

Clocking or indexing is known as a possible method to increase the performance and to reduce the unsteady blade loading in multi-stage turbomachinery using the beneficial aspects of blade row interactions. Using this method, the relative positions

\footnotetext{
* Corresponding author: School of Energy and Power Engineering, Xi'an Jiaotong University, 710049 Xi'an, People's Republic of China. email:xiguang@mail.xjtu.edu.cn
}

of the stator blade rows (and/or rotor blade rows) are altered. Maximum effects can be expected if the blade numbers of the adjacent blade rows are identical.

In the past several decades, many researchers experimentally and numerically investigated the effect of clocking on the efficiency.

Dorney et al. [1] carried out the investigation of clocking on performance in a 1.5-stage compressor. The numerical results show that the improvement in efficiency with stator clocking is $0.6-0.7$ per cent. Maximal efficiency is observed when clocked wake segments of the upstream stator blade row impinge the blade leading edge (LE) of the downstream stator blade. Vice versa, the lowest efficiency is associated with clocked wake segments following a midvane path.

The numerical results in a three-stage low-pressure turbine of Arnone et al. [2] show similar trends as reported by Dorney et al. [1]. The effect of full-clocking (clocking of both the rotor and stator blade rows) on the maximal efficiency variation was estimated to be 0.7 per cent. 
Experimental investigations of stator clocking on performance in a four-stage low-speed axial compressor were performed by Barankiewicz and Hathaway [3]. The results of their investigation show that the change of the overall efficiency due to stator clocking is 0.2 per cent for two operating conditions (peak pressure and peak efficiency conditions). It is considered that the impact of stator indexing on compressor performance is small and in the order of that due to manufacturing and assembly tolerances for this test compressor.

Further investigations on the effect of clocking on the performance were done by Reinmoeller et al. [4], Arnone et al. [5], and Bohn et al. [6].

Besides efficiency, the unsteady profile pressure distribution is significantly affected by clocking of the blade rows. The numerical results performed by Cizmas and Dorney [7] show, for both a compressor and a turbine analysed, that the amplitude of unsteady pressure increases when efficiency increases, i.e. the unsteadiness of the pressure and the blade pressure forces on the rotor and stator blades is maximal at the maximal efficiency clocking position, while minimal at the minimal efficiency clocking position.

Hsu and Wo [8] performed the experimental investigations of the effect of clocking on the unsteady blade loading in a large-scale, low-speed axial compressor rig. They found a 60 per cent reduction in the stator unsteady force when clocking the downstream rotor for a rotor/stator/rotor configuration. For the same compressor, Lee and Feng [9] performed a numerical investigation.

The influence of vane clocking on both the steady and unsteady pressure loadings was experimentally investigated by Haldeman et al. $[\mathbf{1 0}]$ in a one and one-half stage high-pressure turbine. Mailach and Vogeler [11] investigated the effect of stator clocking on unsteady pressure fluctuations on the rotor blades. In a 1.5-stage low-speed axial compressor, the effect of stator clocking on the unsteady pressure fluctuation and the blade pressure force of the rotor blades was numerically carried out by Jia and Vogeler [12].

In the previous literature, most of the experimental and numerical investigations are focused on the operating condition at design point. Investigations on high-loading case were only performed by Hsu and Wo [8] and Dorney et al. [13].

In the current study, the clocking effects of the stator blade rows in the first stage of the low-speed research compressor (LSRC) at an operating point near the stability limit are investigated ( 85 per cent mass flow at design speed: $\xi=0.85, \zeta=1.0$ ). Furthermore selected results at design point $(\xi=1.00, \zeta=1.0)$ are presented for comparison (detailed investigation results at design point for the same compressor can be found in reference [12]).
The investigations are focused on the clocking effects on the profile pressure distribution and blade pressure forces at midspan (MS) of the rotor blades in the first stage of the compressor.

\section{NUMERICAL METHOD, EXPERIMENTAL SET-UP, AND DATA POSTPROCESSING}

The Dresden LSRC consists of four repeating stages which are preceded by an inlet guide vane row (IGV). The blading of the compressor was developed from a typical middle stage of a high-pressure compressor of a gas turbine. A detailed description of the Dresden LSRC is given by Sauer et al. [14], Müller et al. [15], and Boos et al. [16].

In this paper, the unsteady flow field of the first stage with IGV clocking of the Dresden LSRC was investigated using the time-accurate, viscous flow solver FINE $^{\mathrm{TM}}$ / Turbo. The simulations were carried out at the MS of the compressor.

The three-dimensional unsteady compressible Reynolds-averaged Navier-Stokes equations written in the cylindrical coordinate system were solved. The mixing length turbulence model of Baldwin and Lomax [17] is adopted. The governing equations are discretized in space using a cell-centred finite-volume scheme. The artificial dissipation model used is the one originally introduced by Jameson et al. [18]. The control equations are advanced by a time-marching scheme using an explicit four-stage Runge-Kutta method.

Local time stepping, implicit residual smoothing, and multi-grids are used to accelerate the computational convergence. The dual time-stepping method proposed by Jameson [19] is used to carry out timeaccurate calculations.

In the experiment, the blade numbers of the rotor and stator blade rows are 63 and 83, respectively. In the simulation, the blade number of the stator blade rows is adjusted to 84 . In this way, the blade ratio between rotor and stator is $3: 4$. The adjustment of the blade numbers will induce the change of the unsteady profile pressure distribution and the resulting pressure force on blades, although the difference here is slight because of the small adjustment ratio 84/83. The effect of the adjustment of the blade numbers on blade forces will not be discussed here, which can be referred in the investigation of Arnone and Pacciani [20].

The computational mesh and the details of the meshes at the LE and the trailing edge (TE) of the rotor at MS are shown in Fig. 1. The total number of grid points is 379635 .

At the inlet boundary, the inlet flow angle and stagnation parameters are imposed. At the outlet boundary, the pitchwise-averaged static pressure is assigned. The direct periodic conditions are applied on the 
(a)

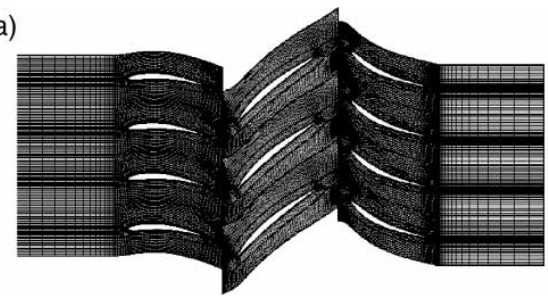

(b)

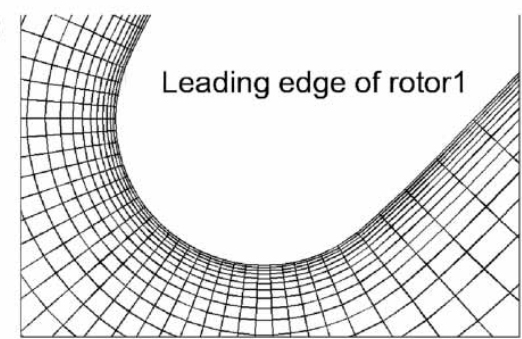

(c)

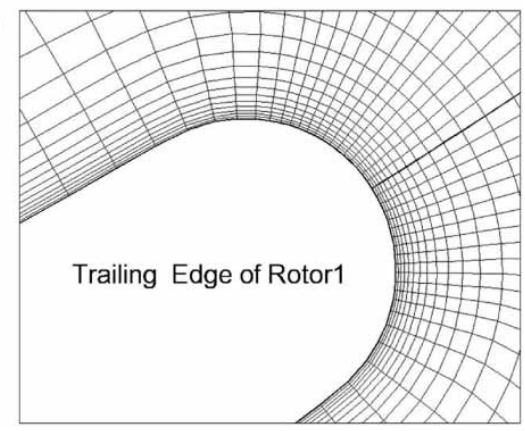

Fig. 1 Computational mesh (zero clocking position)

lower periodic boundary of the first passage and the upper boundary of the last passage. The shroud and hub walls are treated as an Euler wall. At the rotorstator interfaces, mixing plane treatment was used for the steady simulations, whereas a direct interpolation on sliding meshes was employed for the unsteady calculations.

In one simulation period, 90 physical time steps are used. One simulation period is related to the time that one rotor passes four stator blades.

The IGV was traversed one blade pitch in the circumferential direction in steps of 10 per cent of the IGV pitch, whereas the circumferential position of the stator is fixed. The unsteady flow field at each different relative circumferential position between IGV and stator was simulated. A schematic view of the IGV clocking positions and the wake of the IGV at two operating points $(\xi=1.0$ and $\xi=0.85)$ is given in Fig. 2 . The calculations were carried out at MS for design point [12] and an operating point near the stability limit of the compressor.

The unsteady blade pressure forces are calculated from the unsteady profile pressure distribution along the whole chord in the simulation. It should be noticed that friction forces on the blades are not considered.

In the experiment, the unsteady pressure was measured by piezoresistive pressure transducers, which

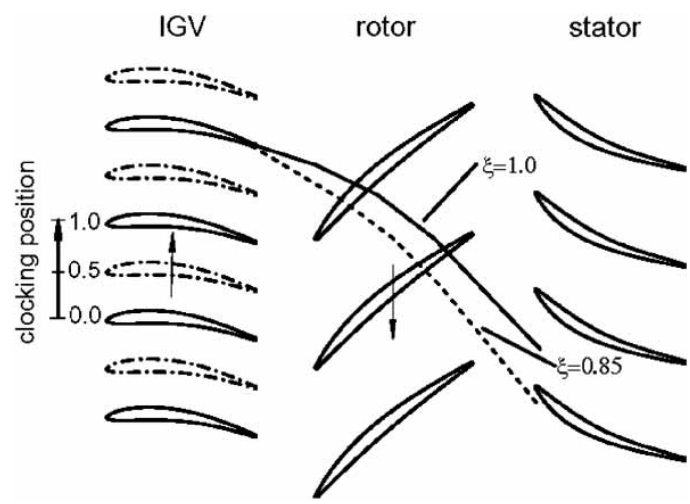

Fig. 2 Schematic view of IGV clocking positions and IGV wake at design point and near stall point

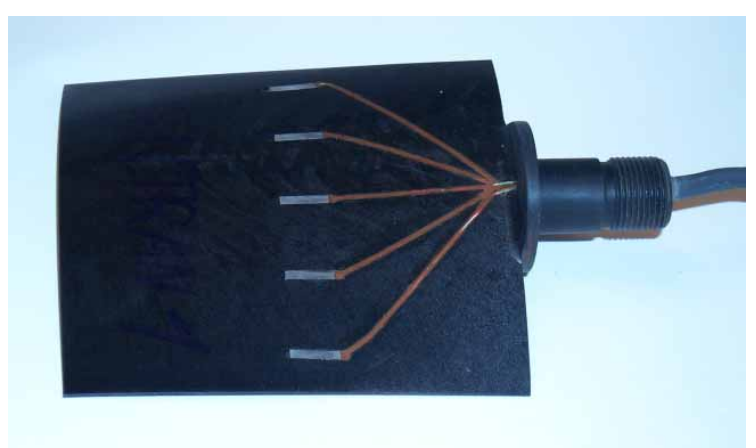

Fig. 3 SS of the rotor blade with embedded piezoresistive pressure transducers

were arranged on the pressure side (PS) and suction side (SS) at MS of one rotor blade. Figure 3 shows the pressure transducers which were equipped on the PS of one rotor blade. From 10 to 90 per cent chord, the transducers are equally distributed by steps of 20 per cent chord. The steady pressure was measured using pressure taps on PS and SS. The pressure at the LE and TE of the blade could not be measured. For the calculation of blade pressure forces, it is extrapolated from the measuring data at the positions next to the LE and TE, respectively.

The algorithm used here to calculate the unsteady pressure force from the experimental and numerical data is described by Mailach et al. [21].

In Fig. 4, the force component definitions in the blade coordinate system are shown. The components of the force $F$ are denoted as $F_{x}$ and $F_{y}$. These components are directed along and perpendicular to the blade chord direction, respectively. The moment $M_{\mathrm{cg}}$ acts around the centre of gravity of the blade.

The force coefficient resulting from the forces in the $x$ - and $y$-directions is calculated with

$$
c_{\mathrm{F}}(t)=\frac{F(t)}{\rho / 2 \cdot \bar{w}_{\infty}^{2} \cdot A}
$$




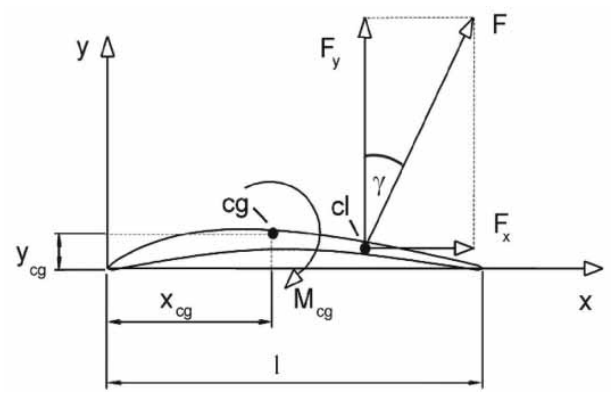

Fig. 4 Schematic view of force definitions in the blade coordinate system

The moment coefficient is calculated with

$$
c_{\mathrm{M}, \mathrm{cg}}(t)=\frac{M_{\mathrm{cg}}(t)}{\rho / 2 \cdot \bar{w}_{\infty}^{2} \cdot A \cdot l}
$$

\section{WAKE DETECTION}

In order to accurately identify the circumferential position of the IGV wake in stator passages, the middle position between two neighbouring LE of the stator blades is selected.

When the wake of the IGV just passes in the middle of the downstream stator blades, the time-averaged entropy value of the middle position, which is induced by the wake of the IGV, should reach its maximum.

It can be observed from Fig. 5 that at clocking position IGV 0.1, the time-averaged entropy value is maximal for design point (solid line with full points), i.e. the wake of IGV passes in the middle of the passage at clocking position IGV 0.1 for design point. The wake of IGV impinges the LE of the downstream stator blades at clocking position IGV 0.6 at this operating point.

At the operating point near the stability limit, the time-averaged entropy value reaches its minimum at

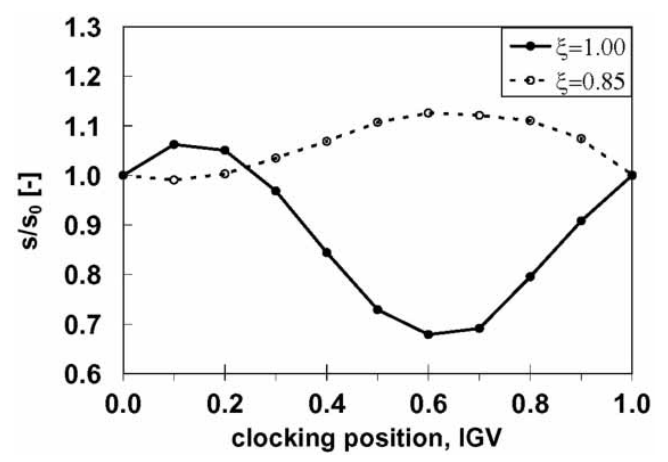

Fig. 5 Time-averaged entropy value at midpitch between two downstream stator-vane LEs versus the IGV clocking positions at MS, for two operating points of reduced mass flow $\xi=1.00$ and $\xi=0.85$ at the reduced speed $\zeta=1.0$ clocking position IGV 0.1, i.e. at the operating point near the stability limit, the wake of the IGV impinges the LE of the downstream stator blades at clocking position IGV 0.1 and passes the middle passage at clocking position IGV 0.6 .

Comparing the results at $\xi=1.0$ with $\xi=0.85$, it can be found that the circumferential position of the IGV wake shifts by 50 per cent stator pitch towards the PS of the stator blades, when the compressor approaches the operating point near the stability limit $(\xi=0.85)$ from design point $(\xi=1.0)$ at design speed. The shift of 50 per cent stator pitch of the wake circumferential position relative to the downstream stator blade is due to the change in the inlet- and the outletflow angle of the rotor blade row and the increase in the turning angle in the rotor blade row.

The wake position of the IGV affects the timeaveraged flow field of the compressor. At different clocking positions, the time-averaged flow field is different. Thus, the wake position of the IGV relative to the LE of the downstream stator has an essential effect on the efficiency of the compressor. In the investigated configuration, the change in the time-averaged flow field at both the design point and the operating point near the stability limit is very small. Therefore, the change in the efficiency with the clocking positions is small and in the range of the accuracy of the numerical simulation. The numerical results are confirmed by the experimental data. In the experiment, the effect of the clocking on the overall performance is in the range of the uncertainty of the experimental equipment at both design point and high-loading case.

\section{TIME-AVERAGED PRESSURE DISTRIBUTION AND PRESSURE FORCES ON THE ROTOR BLADES}

Jia and Vogeler [12] discussed in detail the effect of clocking on time-averaged pressure distribution and pressure forces at design point. It is found that the effect of clocking on the time-averaged pressure distribution is very small, and the difference of timeaveraged pressure forces between different clocking positions cannot be detected.

The investigation results at the operating point near the stability limit show that the clocking effect on the time-averaged pressure distribution and the time-averaged blade pressure force at high loading condition is also very small and the difference of the time-averaged pressure distribution and blade pressure force parameters between different clocking positions is within the range of the simulation accuracy.

Therefore, in Fig. 6 and Table 1, the time-averaged pressure distribution and pressure force parameters only at zero clocking position are given. 


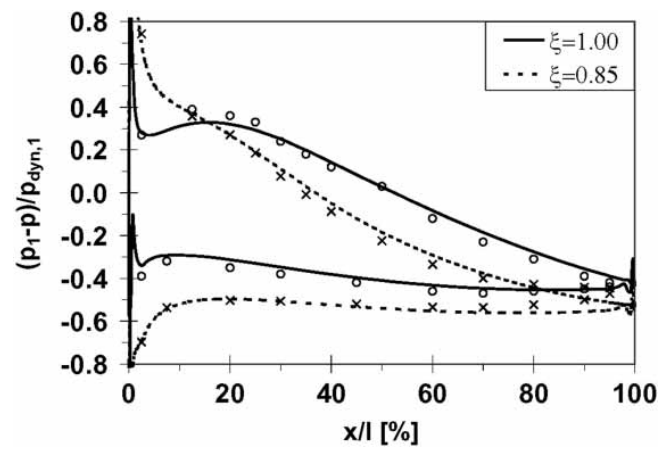

Fig. 6 Time-averaged pressure distribution on the rotor blades at zero clocking position, MS, design speed $(\zeta=1.0)$

Table 1 Time-averaged pressure force parameters on the rotor blades, MS, design speed $(\zeta=1.0)$

\begin{tabular}{llllll}
\hline$\xi$ & $\bar{C}_{\mathrm{F}}$ & $\bar{C}_{\mathrm{F}, y}$ & $\bar{C}_{\mathrm{F}, x}$ & $\bar{C}_{\mathrm{M}, \mathrm{cg}}$ & $\bar{\gamma}[\mathrm{deg}]$ \\
\hline 1.00 & 0.57 & 0.56 & -0.06 & 0.58 & -5.9 \\
0.85 & 0.75 & 0.74 & -0.13 & 1.33 & -9.9 \\
\hline
\end{tabular}

The time-averaged profile pressure distribution at the MS of the rotor blades at two operating conditions at zero clocking position is shown in Fig. 6 . The solid and dashed lines are the numerical results at the design point and the operating point near the stability limit, respectively. The circles and crosses show the experimental data for the two operating points for comparison.

It can be observed that the blade loading increases when the compressor approaches the operating point near the stability limit from design point. The pressure minimum shifts towards the LE of the rotor blades.

On SS, the pressure minimum is close to the LE at the operating point near the stability limit. At design point, the flow accelerates to 15 per cent chord on the SS and from there the flow decelerates to the TE of the blade. At 15 per cent chord, the pressure is minimal.

On PS, the flow decelerates from the LE to 80 per cent chord at design point. From 80 per cent chord towards the TE, the flow accelerates slightly. At the operating point near the stability limit, the pressure gradient from 10 per cent chord to the TE is smaller than that at design point.

The blade pressure force is calculated by integrating the profile pressure distribution with respect to the blade contour. In Table 1, the time-averaged blade pressure force parameters on the rotor blades at two operating conditions are given. At both operating points, the dominating force component on the rotor blades is $F_{y}$ (Table 1 ). The force component coefficient in the $y$-direction is about six to ten times as that in the $x$-direction. Because of the smaller value of the force component coefficient in the $x$-direction
$\left(c_{\mathrm{F}, x}\right)$, the force coefficient $c_{\mathrm{F}}$ is nearly equal to the force component coefficient in the $y$-direction $\left(c_{\mathrm{F}, y}\right)$.

The force coefficient $c_{\mathrm{F}}$ and the dominating force component coefficient $c_{\mathrm{F}, y}$ at the operating point near the stability limit are higher than those at the design point. With the increase in the blade loading, the angle $\gamma$ between the $y$-direction force component and the resulting force increases.

\section{UNSTEADY PRESSURE FLUCTUATIONS ON THE ROTOR BLADES}

Further on, the results at two main clocking positions (IGV 0.0 and IGV 0.6) will be discussed. At these two clocking positions, the fluctuation amplitude of the unsteady loading on the rotor blades reaches its minimum and maximum, respectively.

The unsteady pressure fluctuation on the rotor blades is influenced by the incoming wake effect of the IGV row and the potential effect of the downstream stator blade row. The unsteady pressure fluctuation on the rotor blades is determined by the superimposed effect of the wake effect and the downstream potential effect.

In order to identify the point of time at which the incoming wake impinges the LE of the rotor blades and the potential effect reaches the TE of the rotor blades, the axial velocity at one investigated point before the LE point and the static pressure at one point behind the TE point are observed. The axial positions of the two observed points are $3 \mathrm{~mm}$ away from the LE and TE points of the considered rotor blade, respectively, whereas the circumferential positions of the two observed points are the same as those of the LE and TE points, respectively. When the incoming wake of the IGV reaches the observed point, the axial velocity should be smaller than that in the main flow.

Figures 7(a) and (b) show the unsteady axial velocity and the unsteady relative flow angle near the LE of the rotor at two operating points $(\xi=1.0$ and $\xi=0.85)$ at clocking position IGV 0.0, whereas in Figs 7(c) and (d), the unsteady axial velocity and the unsteady relative flow angel at clocking position IGV 0.6 are given for the two operating points.

In Fig. 7 , time ( $x$-axis) is related to the stator blade passing period $t_{\text {stator }}$.

In Figs 7(b) and (d), relative flow angle is defined as the angle against the circumferential direction.

In Figs 7(b) and (d), the value of the $y$-axis at left shows the flow angle at design point $(\xi=1.0)$ and the right $y$-axis gives the flow angle at the operating point near the stability limit $(\xi=0.85)$.

It can be seen in Figs 7(a) and (c) that the depth and width of the wake are nearly the same when the operating condition of the compressor is changed from the design point to the operating point near the stability 

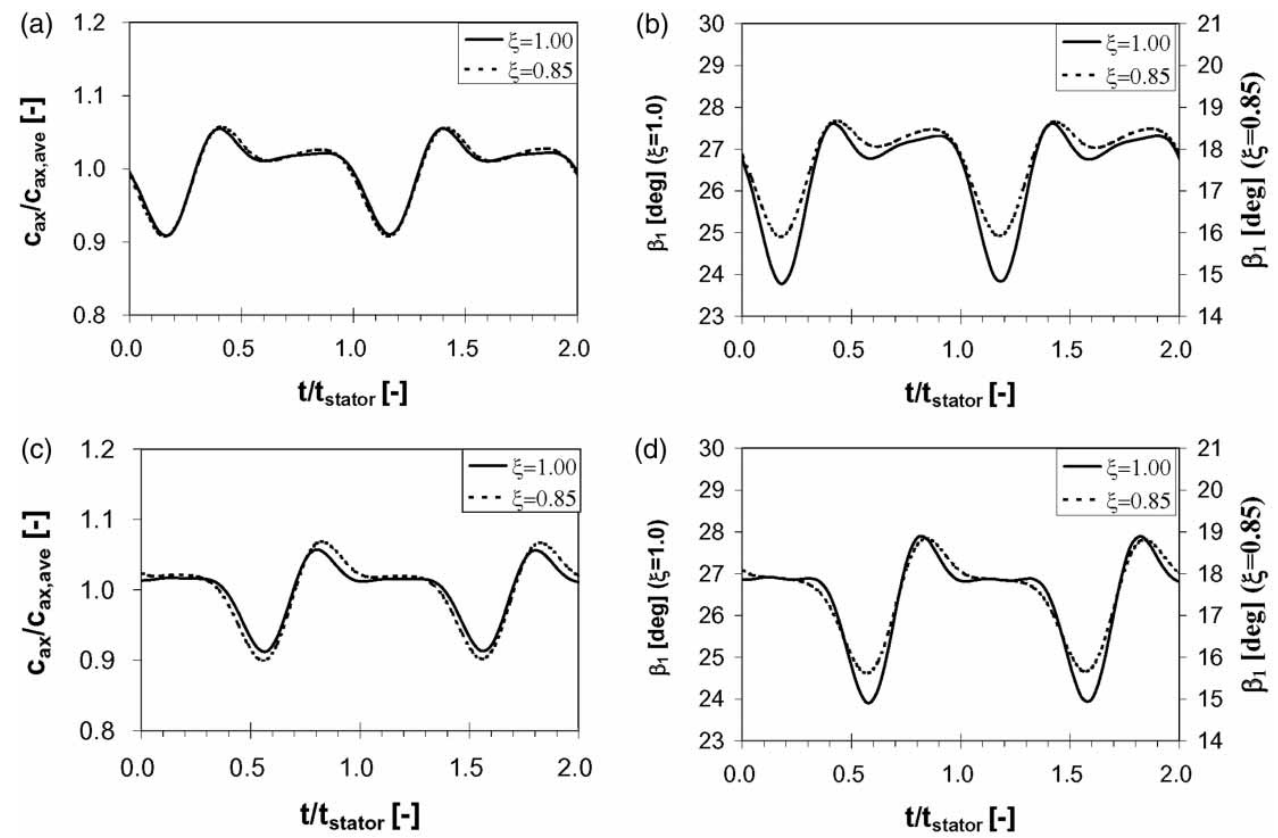

Fig. 7 Unsteady axial velocity and relative flow angle $3 \mathrm{~mm}$ upstream of the rotor blade LE, MS, design speed $(\zeta=1.0$ ): (a) clocking position IGV 0.0, axial velocity and (b) clocking position IGV 0.0, inlet flow angle, (c) clocking position IGV 0.6, axial velocity, and (d) clocking position IGV 0.6, inlet flow angle

limit. However, the relative inlet flow angle versus the circumferential direction decreases with the change inthe operating points from $\xi=1.0$ to $\xi=0.85$. At the investigated position just $3 \mathrm{~mm}$ upstream of the LE of the rotor blade, the relative inlet flow angle at $\xi=1.0$ is $26^{\circ}$, while at $\xi=0.85$, the flow angle decreases to $18^{\circ}$. Thus, the blade loading on the rotor blades increases when the mass flow is reduced.

At the investigated position just $3 \mathrm{~mm}$ upstream of the LE of the rotor blade, the maximal fluctuation of the inlet flow angle is induced by the outgoing flow of the IGV and the viscous wakes of the IGV. The effect of the potential field of the IGV is secondary. At every single clocking position, the passing time of the IGV can be clearly observed with the steep decrease in the axial velocity and the relative flow angle at the inlet of the rotor.

It can be observed in Figs 7(b) and (d) that the maximal fluctuation amplitude of the relative inlet flow angle at $\xi=0.85$ is smaller than that at $\xi=1.0$ at both clocking positions, IGV 0.0 and IGV 0.6.

For the potential effect of the downstream stator blade row, it is difficult to distinguish the point in time for a single clocking position. Thus, in Fig. 8, the results at ten clocking positions are given.

In Figs 8(a) and (c), the space-time contour of the unsteady static pressure near the TE of the rotor blade is shown for $\xi=1.0$ and $\xi=0.85$, respectively. In Figs 8 (b) and (d), the unsteady relative flow angle at the rotor outlet is presented for the two operating points at the same position. The $x$-axis is the time related to the stator blade passing period $t_{\text {stator }}$. The $y$-axis is the clocking position of the IGV.

When the potential effect reaches the investigated position, the static pressure should be larger than that at the other points of time. When the wake from the IGV arrives at the observed point, both the velocity and the static pressure should be smaller.

In the investigation of the clocking effect, the position of the IGV is traversed in the circumferential direction in steps of 10 per cent of the IGV pitch, whereas the stator is fixed. Thus, the effect of the IGV wake appears at the TE of the rotor at different points of time at different clocking positions, whereas the potential effect appears at the same point in time. The effect of a lowered static pressure due to the IGV wake is marked with white dashed lines and the potential effect as a maximum of the static pressure is pointed out with arrows.

When Fig. 8(a) is compared with Fig. 8(c), it can be noticed that the wake effect at both $\xi=1.0$ and $\xi=0.85$ appears at the same point in time $\left(t / t_{\text {stator }}=\right.$ $0.4,1.4, \ldots$ at clocking position IGV 0.0 ). The point in time, at which the potential effect of the downstream stator reaches the considered point, changes a little between the different operating points. It can be found at $t / t_{\text {stator }}=0.8,1.8, \ldots$ for design point and at $t / t_{\text {stator }}=0.85,1.85, \ldots$ for the operating point near the stability limit. 

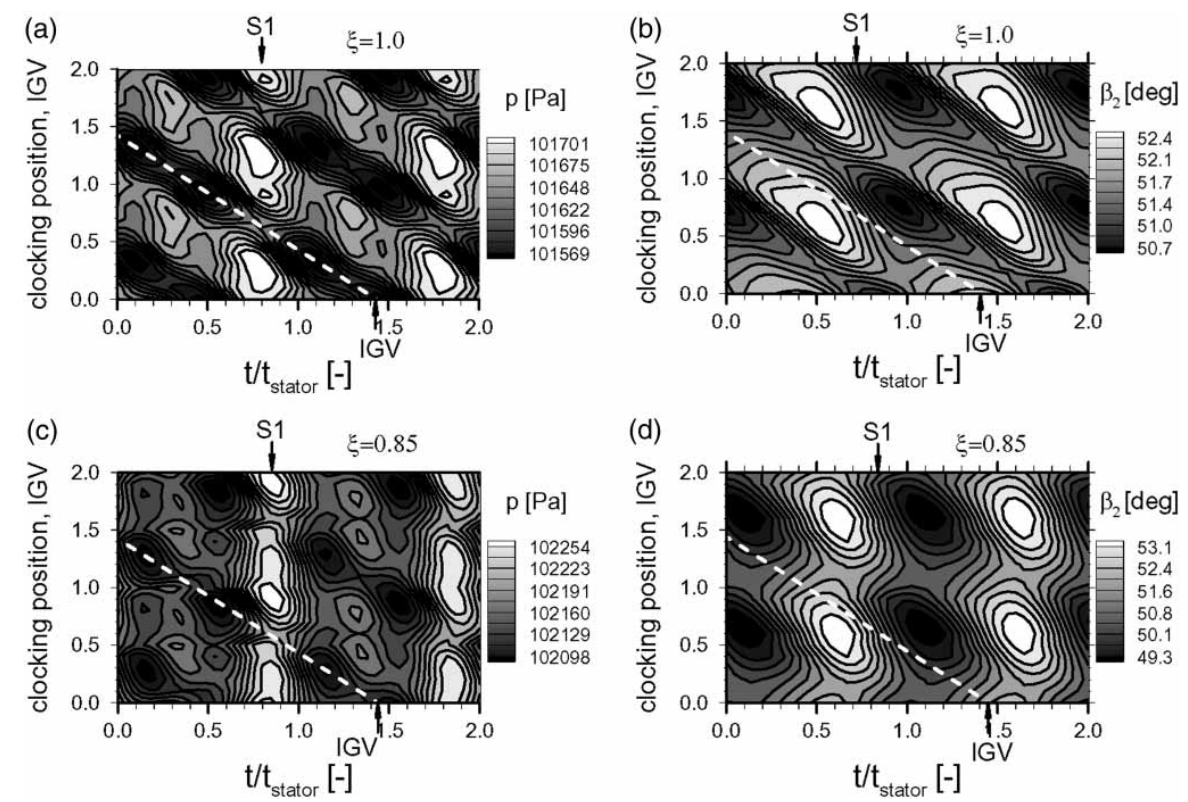

Fig. 8 Unsteady static pressure (a and c) and relative outlet flow angle (b and d) versus the IGV clocking positions at $3 \mathrm{~mm}$ downstream of the rotor TE, MS, design speed $\zeta=1.0$

The unsteady relative flow angles for all clocking positions at two operating points are presented in Figs $8(b)$ and (d), respectively. The white dashed line is the point in time of the wake effect obtained according to the results of the static pressure in Figs 8(a) and (c).

On comparing Fig. 8(b) with Fig. 8(d), it can be observed that the fluctuation in the relative outlet flow angle at the operating point $\xi=0.85$ is stronger than that at design point at both clocking positions IGV 0.0 and IGV 0.6. Especially at clocking position IGV 0.6, the increase in the fluctuation amplitude from $\xi=1.0$ to $\xi=0.85$ is more obvious.

In order to observe the change of the unsteady pressure on PS and SS of the rotor blades at different points in time, the space-time contour of the unsteady pressure fluctuation at clocking positions IGV 0.0 and IGV 0.6 at two operating points $(\xi=1.0$ and $\xi=0.85$ ) is shown in Figs 9 and 10.

Because of the identical blade numbers of the IGV and stator blade row, the wake effect and the potential
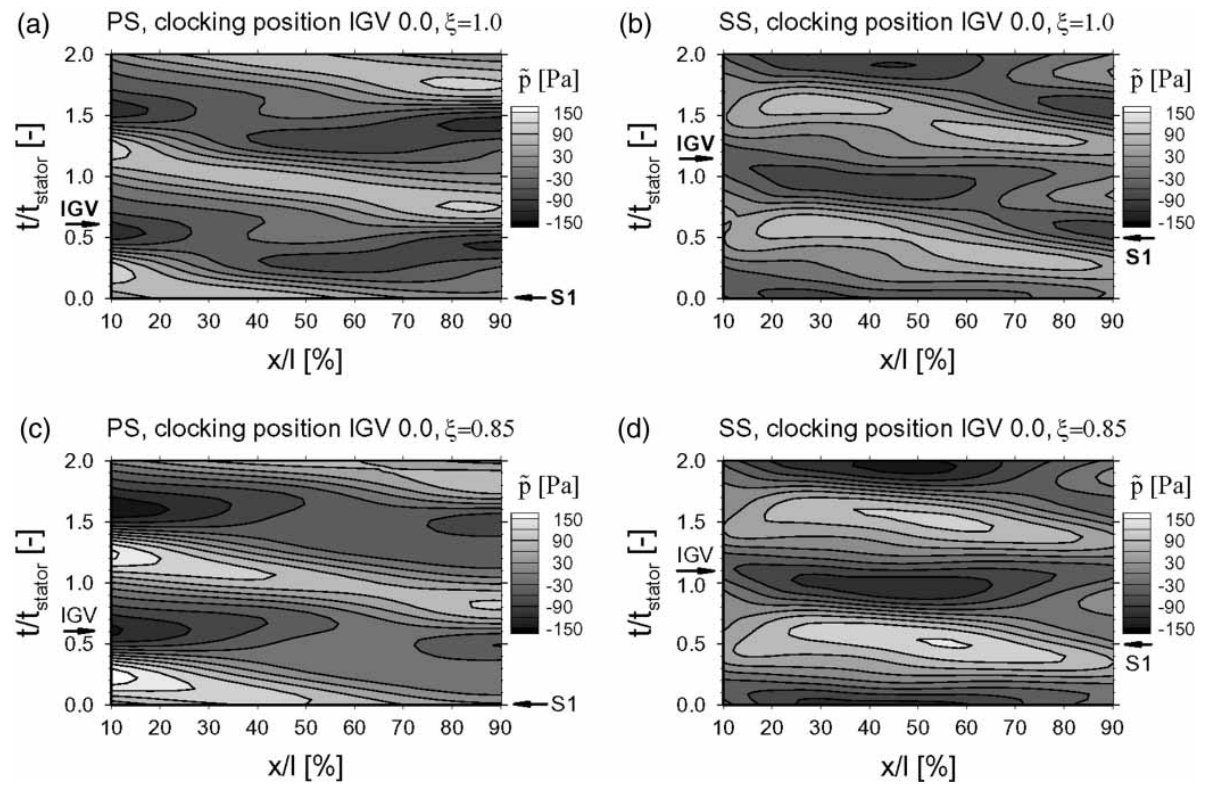

Fig. 9 Unsteady pressure fluctuations on PS (a and c) and SS (b and d) of the rotor blades, MS, clocking position IGV 0.0, design speed $\zeta=1.0$ 

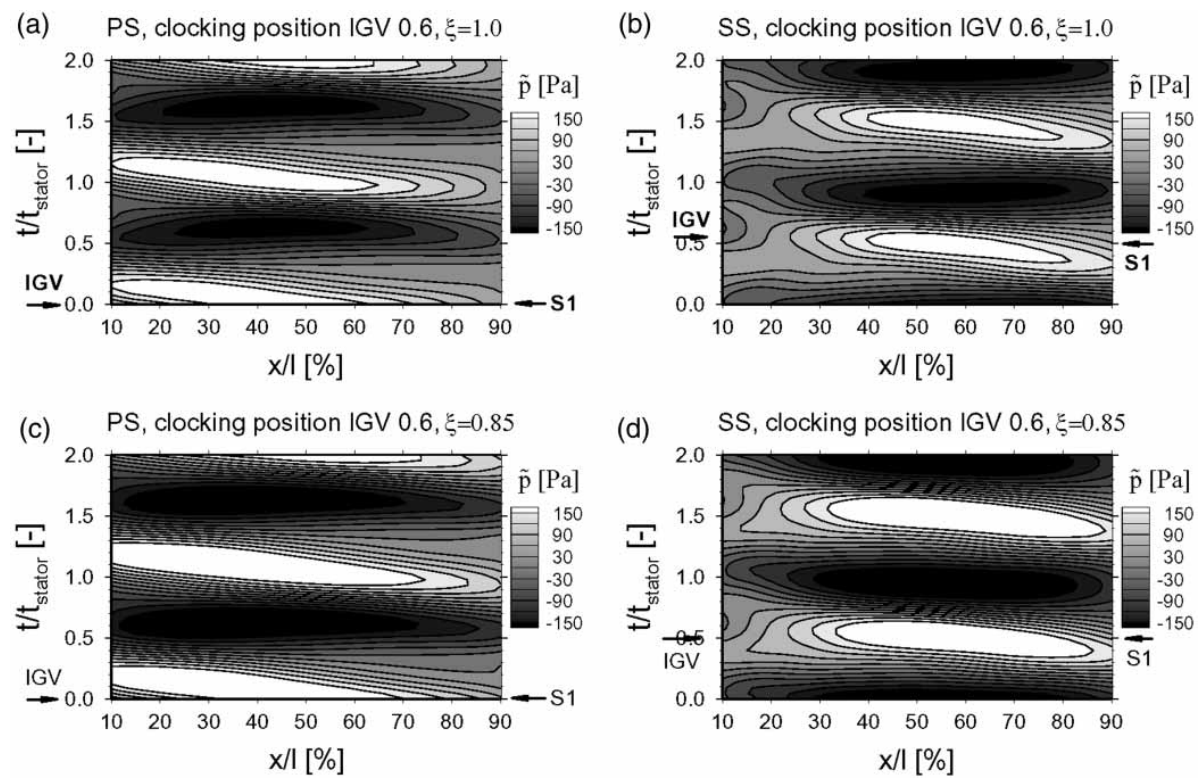

Fig. 10 Unsteady pressure fluctuations on PS (a and c) and SS (b and d) of the rotor blades, MS, clocking position IGV 0.6, design speed $\zeta=1.0$

effect of the downstream stator blade row cannot be distinguished in the unsteady pressure fluctuation for a single clocking position. In the space-time contour of the unsteady pressure fluctuation at one considered chord length for different clocking positions, the point in time of the two different effects (the wake effect of the IGV and the potential effect of the downstream stator blade row) can be determined. The detailed discussion can be found in reference [12] for the design point of the same compressor.

Because the unsteady pressure fluctuation on the rotor blades changes nearly simultaneously along the whole blade chord [11, 22], it can be assumed that the effect of the IGV wake and the potential effect appear nearly at the same point in time along the whole blade chord, respectively.

Figures 9 and 10 show the unsteady pressure fluctuation on the PS and SS of the rotor blades along the chord length for the design point and the operating point near the stability limit at clocking position IGV 0.0 and IGV 0.6, respectively. At these two clocking positions, the fluctuation amplitude of the unsteady blade loading on the rotor blades reaches its minimum and maximum among the investigated ten different clocking positions, respectively.

The $x$-axis in Figs 9 and 10 is the non-dimensional chord. The time is related to the passing period of the downstream stator $t_{\text {stator }}$.

In Figs 9 and 10, the point in time of the IGV wake effect and the potential effect on the unsteady pressure fluctuation are marked with arrows, which is identified according to the space-time diagram of the unsteady pressure fluctuation on PS and SS at 50 per cent chord with clocking positions (the figures in reference [12] for design point, no figure for the operating point near the stability limit).

According to Figs 7 and 8 , it can be seen that the wake effect of the IGV and the potential effect of the downstream stator blade row appear nearly at the same point in time for two different operating conditions $(\xi=1.0$ and $\xi=0.85)$. Thus, the change in the fluctuation amplitude of the unsteady pressure on the rotor blades with clocking positions is similar for different operating points.

The maximal pressure fluctuation at clocking position IGV 0.0 for both, design point and operating point near the stability limit, is much lower than that at clocking position IGV 0.6 (compare Fig. 9 with Fig. 10). The reason is that the pressure fluctuation on the rotor blades induced by the IGV wake and the pressure fluctuations induced by the potential effect of the downstream stator blades are superimposed with approximately opposite signs at clocking position IGV 0.0 . At clocking position IGV 0.6 , the pressure fluctuation induced by the IGV wake and the potential effect of the downstream stator blades appears nearly at the same point in time. Thus, the fluctuation amplitude increases.

The unsteady pressure fluctuation on the rotor blades reappears periodically at every stator blade passing period $t_{\text {stator }}$ because of the identical blade numbers of the IGV and the stator blade row for all clocking positions and for all operating conditions.

The fluctuation amplitude of the unsteady pressure at the operating point near the stability limit at every clocking position is somewhat higher than that 
at the corresponding clocking position at design point (compare Figs 9(a) and (b) with Figs 9(c) and (d), and Figs 10(a) and (b) with Figs 10(c) and (d)).

At clocking positions IGV 0.0 and IGV 0.6, the maximal fluctuation amplitude of the unsteady pressure at design point is \pm 3 and \pm 8 per cent of the dynamic head of the incoming flow, respectively. At the operating point near the stability limit, it is about \pm 5 and \pm 10 per cent of the dynamic head, respectively, i.e. the unsteady pressure fluctuation can be damped and amplified by the clocking of IGV, which can also be proved in the investigation of the blade pressure force at different clocking positions in the next section.

According to the above discussion, one can recognize that the clocking position with maximal or minimal unsteady loading is hardly shifted with the change in the operating conditions. The reason can be explained using the following simple model.

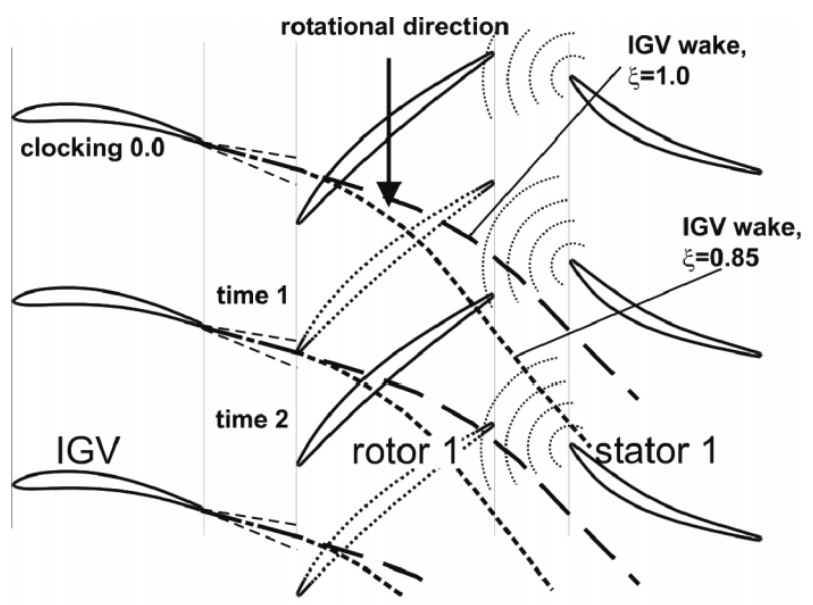

(a)

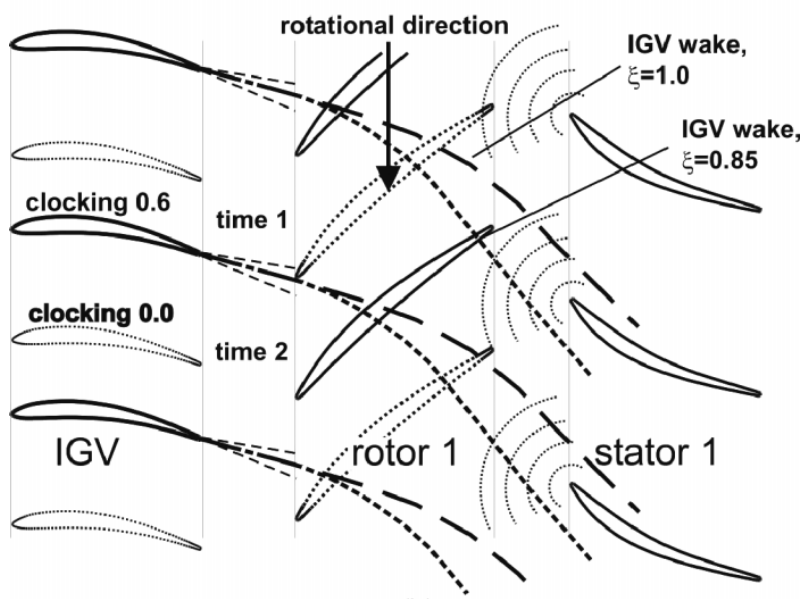

(b)

Fig. 11 Schematic view of the interaction mechanism
In Fig. 11(a), the interaction process at clocking position IGV 0.0 for two operating points is shown. The instantaneous position of the rotor blade row at two points of time is presented. At time 1, the wake impinges the LE of the rotor blade, whereas the TE of the rotor blade locates in the middle of the potential effect. At time 2, the potential effect reaches the TE of the rotor blade, whereas the LE of the rotor blade is situated in the middle of the wake effect. The interaction process at clocking position IGV 0.0 is nearly the same for two different operating points. The reason is that, on the one hand, the position and the characteristic of the incoming IGV-wake for two operating conditions do not change at the LE of the rotor blades, although the position of the wake shifts 50 per cent stator pitch at the LE of the downstream stator blades due to the increased loading. This is clearly verified in Fig. 7. On the other hand, the potential effect reaches the TE of the rotor blades also at nearly the same point in time for different operating points. By comparing the flow parameters shown in Fig. 8 with the two operating points, one can recognize that the behaviour of the static pressure as well as the relative flow angle near the TE of the rotor differs only marginally between the two operating points.

At clocking-position IGV 0.6 (Fig. 11(b)), the interaction process is as follows: at time 1, the LE of rotor 1 is located within the wake, shed by the IGV. At the same time the TE of rotor 1 is influenced by the potential effect from the downstream stator 1 . Thus, the loading at both LE and TE of rotor 1 is increased. At time 2, the LE of rotor 1 is situated in the middle between two IGV-wakes. The TE of rotor 1 is also at a circumferential position between the potential effects of two downstream stator blades. Therefore, the difference between the low loading at both LE and TE at time 2 and the high loading at time 1 is remarkably larger than for clocking position IGV 0.0 (Fig. 11(a)).

For a given clocking position (e.g. IGV 0.6), the circumferential position of the IGV-wake, relative to the leading edges of the stator blades, shifts by 50 per cent of the stator pitch from the design point to the operating point near the stability limit, due to the difference in the rotor's turning angle. This is, however, not a dominating effect on the unsteady blade loading of rotor 1. In spite of this, the IGV's wake reaches the LE of rotor 1 at nearly the same point of time in both operating conditions (Figs 7(c) and (d)). This is mainly due to negligible changes of the outflow angles of the IGV at different operating points. The shape of the potential flow field of the downstream stator 1 shows only small changes in its circumferential distribution at the two operating points (Fig. 8). Thus, the superimposed effects of the IGV-wake and the potential field of stator 1 , as well as the fluctuation amplitude of the pressure 


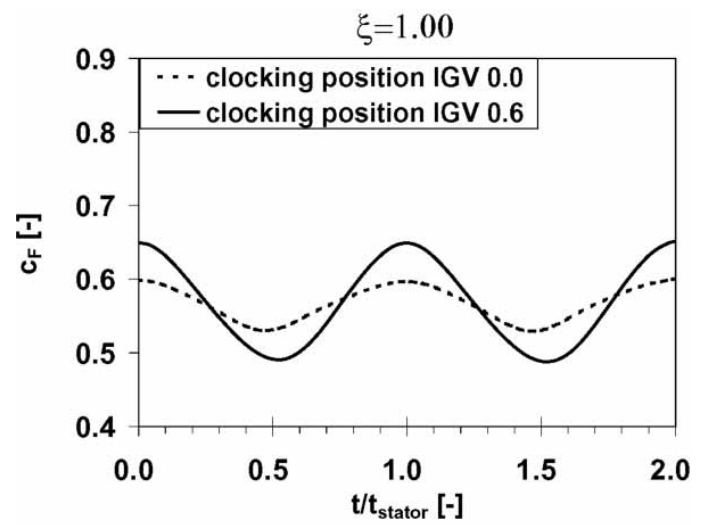

(a)

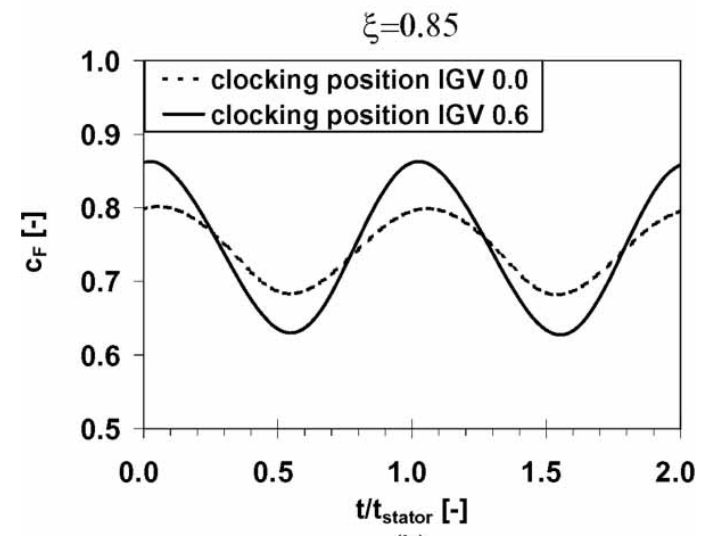

(b)

Fig. 12 Unsteady pressure force on the rotor blades, MS, design speed $(\zeta=1.0)$

forces on the rotor blades, are nearly the same for both operating points.

\section{UNSTEADY PRESSURE FORCES ON THE ROTOR BLADES}

According to the unsteady pressure distribution profile, the unsteady blade pressure force can be calculated. The blade pressure force is not only dependent on the amplitude of the unsteady pressure, but also on the phase shift between signals on PS and SS.

Figure 12 shows the unsteady pressure force at two clocking positions at the operating point near the stability limit $(\xi=0.85)$. The results of the unsteady pressure force at design point can be referred in reference $[12]$. The time is related to the stator blade passing period $t_{\text {stator }}$. At the two clocking positions IGV 0.0 and IGV 0.6, the fluctuation amplitudes on the rotor blades reach the minimum and maximum, respectively.

The pressure force changes periodically in every stator passing period $t_{\text {stator }}$ because of the identical blade numbers of the IGV and the stator blades. It can clearly be seen that the fluctuation amplitude at clocking
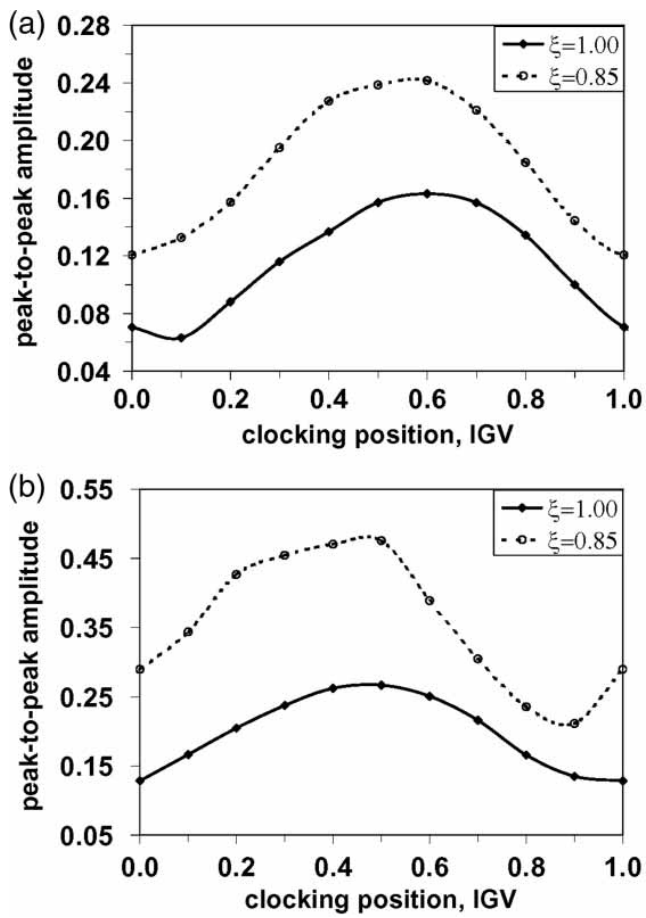

Fig. 13 Peak-to-peak amplitude of the rotor blade pressure forces depending on the clocking position of the IGV, MS, design speed $(\zeta=1.0$ ): (a) simulation and (b) experiment

position IGV 0.0 is lower than that at clocking position IGV 0.6. The peak-to-peak amplitudes at the two clocking positions in Fig. 12 are identical with the values in Fig. 13(a) for the corresponding clocking positions at $\xi=0.85$, respectively.

Figure 13 shows the peak-to-peak amplitude of the unsteady pressure force coefficient $c_{\mathrm{F}}$ at the MS of the rotor blades for different clocking positions at two operating conditions $(\xi=1.0$ and $\xi=0.85)$. Figure 13(a) shows the numerical results and Fig. 13(b) the experimental ones.

It can be observed that the graph of the peak-to-peak amplitude with the clocking position of IGV between two different operating conditions is very similar in both the numerical and experimental results.

In the simulation results (Fig. 13(a)), the maximal and minimal fluctuation amplitudes of the unsteady rotor loading at design point appear at clocking positions IGV0.6 and IGV 0.1, respectively. At the operating point near the stability limit, the clocking positions with the maximal and minimal fluctuation amplitudes appear at clocking positions IGV 0.6 and IGV 0.0 , respectively, i.e. the clocking positions with the maximal and minimal fluctuation amplitudes at the operating point near the stability limit shift about 0 10 per cent stator pitch relative to those at design point.

Similarly, in the experimental results (Fig. 13(b)), the maximal and minimal fluctuation amplitudes appear 
nearly at the same clocking positions at both design point (clocking position IGV 0.5 with maximal amplitude, clocking position IGV 0.0 with minimal amplitude) and the operating point near the stability limit (clocking positions IGV 0.5 and IGV 0.9, respectively).

With the increase in the rotor blade loading at high-loading case, the maximal fluctuation amplitude increases at the corresponding clocking position for both the numerical and experimental results.

In the simulation, at design point $(\xi=1.0)$, the minimal fluctuation amplitude is \pm 6 per cent of the timeaveraged value at clocking position IGV 0.1, whereas the maximal fluctuation amplitude is \pm 14 per cent of the mean value at clocking position IGV 0.6. At the operating point near the stability limit $(\xi=0.85)$, the minimal and maximal fluctuation amplitudes are \pm 8 per cent at clocking position IGV 0.0 and \pm 16 per cent of the mean value at clocking position IGV 0.6, respectively.

In the experiment, the fluctuation amplitude of the unsteady loading on the rotor blades is higher than that in the simulation, which can also be concluded from the comparison between the simulation and the experiment in references [23] and [24].

The difference of the clocking positions with the maximal and minimal amplitudes between the simulation and the experiment is only 10 per cent stator pitch.

According to Fig. 13, it can be calculated that the maximal fluctuation amplitude of the unsteady loading can be reduced 50 per cent at high-loading case and about 60 per cent at design point with the IGV clocking in the simulation, whereas in the experiment, the maximal fluctuation amplitude of the unsteady loading at both the design point and the operating point near the stability limit can be reduced by about 55 per cent.

\section{SUMMARY}

The clocking effect of the IGV row in the LSRC Dresden is numerically investigated. The results at a design point and an operating point near the stability limit are compared and discussed.

The clocking effects on the time-averaged pressure distribution and the time-averaged pressure force of the rotor blades were found to be very small at both the operating point near the stability limit and the design point. However, the unsteady pressure fluctuation and the unsteady pressure force on the rotor blades are significantly influenced by the clocking of the stator blades.

The unsteady blade loading can be reduced remarkably (50-60 per cent reduction in comparison to the maximum loading achievable) by the clocking of the stator blade row at both the operating point near the stability limit and the design point. In addition, the clocking position with the minimal unsteady rotor loading is scarcely dependent on operating points in the investigated LSRC Dresden. The adaptable operating range is from a design point $(\xi=1.0)$ to an operating point near the stability limit with 85 per cent mass flow $(\xi=0.85)$ at design speed. At both the operating point near the stability limit and the design point, the minimal fluctuation amplitude of the unsteady pressure force on the rotor blades appears nearly at the same clocking position for the compressor.

\section{ACKNOWLEDGEMENTS}

The first author would like to express her appreciation to DAAD (German Academic Exchange Service) for the scholarship support. The experimental data are from the project 'Unsteady effects of clocking of stator blade rows in a low-speed axial compressor' funded by the DFG (German Research Society).

\section{REFERENCES}

1 Dorney, D. J., Sharma, O. P., and Gundy-Burlet, K. L. Physics of airfoil clocking in a high-speed axial compressor. ASME paper 98-GT-082, 1998.

2 Arnone, A., Marconcini, M., Pacciani, R., Schipani, C., and Spano, E. Numerical investigation of airfoil clocking in a three-stage low-pressure turbine. ASME, J. Turbomach., 2002, 124(1), 61-68.

3 Barankiewicz, W. S. and Hathaway, M. D. Effects of stator indexing on performance in a low speed multistage axial compressor. ASME paper 97-GT-496, 1997.

4 Reinmoeller, U., Stephan, B., Schmidt, S., and Niehuis, R. Clocking effects in a 1.5 stage axial turbinesteady and unsteady experimental investigations supported by numerical simulations. ASME, J. Turbomach., 2002, 124(1), 52-60.

5 Arnone, A., Marconcini, M., Greco, A. S. D., and Spano, E. Numerical investigation of three-dimensional clocking effects in a low pressure turbine. ASME, J. Turbomach., 2004, 126(3), 375-384.

6 Bohn, D., Ren, J., and Sell, M. Influence of stator clocking on the unsteady three-dimensional flow in a two-stage turbine. ASME, J. Turbomach., 2005, 127(1), 156-163.

7 Cizmas, P. G. A. and Dorney, D. J. The influence of clocking on unsteady forces of compressor and turbine blades. ISABE paper 99-7231, 1999.

8 Hsu, S. T. and Wo, A. M. Reduction of unsteady blade loading by beneficial use of vortical and potential disturbances in an axial compressor with rotor clocking. ASME, J. Turbomach., 1998, 120(4), 705-713.

9 Lee, Y. and Feng, J. Potential and viscous interactions for a multi-blade-row compressor. ASME, J. Turbomach., 2004, 126(4), 464-472.

10 Haldeman, C. W., Krumanaker, M. L., and Dunn, M. G. Influence of clocking and vane/blade spacing on the unsteady surface pressure loading for a modern stage 
and one-half transonic turbine. ASME, J. Turbomach., 2003, 125(4), 743-753.

11 Mailach, R. and Vogeler, K. Rotor-stator interactions in a four-stage low-speed axial compressor - part I: unsteady profile pressures and the effect of clocking. ASME, $J$. Turbomach., 2004, 126(4), 507-518.

12 Jia, H. X. and Vogeler, K. Effect of clocking on the unsteady rotor blade loading in a 1.5-stage low-speed axial compressor. ASME paper GT2007-27237, 2007.

13 Dorney, D. J., Sondak, D. L., Cizmas, P. G. A., Saren, V. E., and Savin, N. M. Full-annulus simulations of airfoil clocking in a compressor at off-design operating conditions. AIAA paper 99-2383, 1999.

14 Sauer, H., Bernstein, W., Bernhard, H., Biesinger, T., Boos, P., and Möckel, H. Konstruktion, Fertigung und Aufbau eines Verdichterprüfstandes und Aufnahme des Versuchsbetriebes an einem NiedergeschwindigkeitsAxialverdichter in Dresden. Abschlußbericht zum BMBFVorhaben 0326758A, Dresden, Germany, 1996.

15 Müller, R., Mailach, R., and Lehmann, I. The design and construction of a four-stage low-speed research compressor. In Proceedings of the IMP 97 Conference on Modelling and Design in Fluid-Flow Machinery, Gdansk, Poland, 1997, pp. 523-530.

16 Boos, P., Möckel, H., Henne, J. M., and Selmeier, R. Flow measurement in a multistage large scale low speed axial flow research compressor. ASME paper 98-GT-432, 1998.

17 Baldwin, B. and Lomax, H. Thin layer approximation and algebraic model for separated turbulent flows. AIAA paper 78-257, 1978.

18 Jameson, A., Schmidt, W., and Turkel, K. E. Numerical solutions of the Euler equations by finite volume methods using Runge-Kutta time-stepping schemes. AIAA paper 81-1259, 1981.

19 Jameson, A. Time dependent calculations using multigrid, with applications to unsteady flows past airfoils and wings. AIAA paper 91-1596, 1991.

20 Arnone, A. and Pacciani, R. Rotor-stator interaction analysis using the Navier-Stokes equations and a multigrid method. ASME, J. Turbomach., 1996, 118(4), 679-689.

21 Mailach, R., Müller, L., and Vogeler, K. Rotor-stator interactions in a four-stage low-speed axial compressor part II: unsteady aerodynamic forces of rotor and stator blades. ASME, J. Turbomach., 2004, 126(4), 519-526.

22 Jia, H. X., Vogeler, K., Müller, L., and Mailach, R. Numerical investigation of rotor-stator interactions in a 1.5stage low-speed axial compressor. In the Conference on Modelling Fluid Flow (CMFF'06), Budapest, Hungary, 2006, pp. 1155-1162.

23 Jia, H. X., Xi, G., Müller, L., Mailach, R., and Vogeler, K. Effect of clocking on unsteady blade loading in a fourstage low-speed axial compressor, part 1: numerical investigation. ISABE paper 2007-1181, 2007.
24 Müller, L., Mailach, R., Vogeler, K., Jia, H. X., and Xi, G. Effect of clocking on unsteady blade loading in a fourstage low-speed axial compressor, part 2: experimental investigations. ISABE paper 2007-1182, 2007.

\section{APPENDIX}

\section{Notation}

A

$c_{\mathrm{ax}} \quad$ axial velocity $(\mathrm{m} / \mathrm{s})$

$c_{\mathrm{F}} \quad$ pressure force coefficient

$c_{\mathrm{M}} \quad$ moment coefficient

$F \quad$ pressure force $(\mathrm{N})$

$l \quad$ chord length $(\mathrm{m})$

$M \quad$ moment (Nm)

$p \quad$ pressure $(\mathrm{Pa})$

$\tilde{p} \quad$ fluctuating part of pressure $(\mathrm{Pa})$

$s \quad$ entropy $(\mathrm{J} / \mathrm{kg} \mathrm{K})$

$t \quad$ time (s)

$w \quad$ relative velocity $(\mathrm{m} / \mathrm{s})$

$x \quad$ chordwise position (m)

$y \quad$ position perpendicular to chord, or circumferential coordinate (m)

$\gamma \quad$ angle between resulting force and normal direction $\left(^{\circ}\right)$

$\zeta \quad$ reduced speed/reduced design speed

$\xi \quad$ reduced mass flow/reduced design mass flow

$\rho \quad$ density $\left(\mathrm{kg} / \mathrm{m}^{3}\right)$

\section{Subscripts and superscripts}

ave average value

cg centre of gravity

cl centre of lift

dyn dynamic

$x \quad$ component in blade chord direction

$y \quad$ component perpendicular to the blade chord time-averaged values

$\sim \quad$ fluctuating part

$\infty \quad$ average of the velocity vectors of the incoming and outgoing flow

$0 \quad$ value for the zero clocking position

1 measuring plane upstream of the rotor blade row

2 measuring plane downstream of the rotor blade row 